\title{
Filigrane
}

Écoutes psychothérapiques

\section{De la diffraction des groupes internes à la reconstruction d'imagos familiales}

\section{Richard Durastante et Christiane Joubert}

Volume 20, numéro 2, automne 2011

URI : https://id.erudit.org/iderudit/1007612ar

DOI : https://doi.org/10.7202/1007612ar

Aller au sommaire du numéro

Éditeur(s)

Revue Santé mentale au Québec

ISSN

1192-1412 (imprimé)

1911-4656 (numérique)

Découvrir la revue

Citer cet article

Durastante, R. \& Joubert, C. (2011). De la diffraction des groupes internes à la reconstruction d'imagos familiales. Filigrane, 20(2), 87-97.

https://doi.org/10.7202/1007612ar

\section{Résumé de l'article}

L'adolescent en souffrance rejoue et diffracte sur l'institution des modalités de liens spécifiques à sa famille interne. Si l'on considère que les groupes internes désignent des schèmes de relations d'objet intériorisés, inhérentes au groupe primaire, l'on peut alors considérer que les groupes externes peuvent être utilisés comme autant de scènes qui permettent de les réactiver. La prise en compte de la manière dont l'adolescent entre en lien avec l'institution pourra éclairer le soignant sur la manière dont il signifie son mal-être en deçà des mots, et dont il rejoue des liens familiaux anciens. Mailler des liens autour de l'adolescent, sur le mode d'une enveloppe familiale contenante, lui permet de reconstruire des imagos familiales satisfaisantes et peu à peu de se différencier et de s'individuer. 


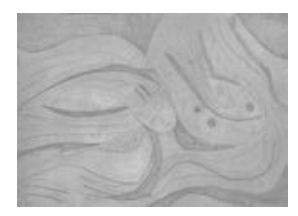

\section{De la diffraction des groupes internes à la reconstruction d'imagos familiales}

\section{Richard Durastante et Christiane Joubert}

L'adolescent en souffrance rejoue et diffracte sur l'institution des modalités de liens spécifiques à sa famille interne. Si l'on considère que les groupes internes désignent des schèmes de relations d'objet intériorisés, inhérentes au groupe primaire, I'on peut alors considérer que les groupes externes peuvent être utilisés comme autant de scènes qui permettent de les réactiver. La prise en compte de la manière dont l'adolescent entre en lien avec l'institution pourra éclairer le soignant sur la manière dont il signifie son mal-être en deçà des mots, et dont il rejoue des liens familiaux anciens. Mailler des liens autour de l'adolescent, sur le mode d'une enveloppe familiale contenante, lui permet de reconstruire des imagos familiales satisfaisantes et peu à peu de se différencier et de s'individuer.

l est possible de travailler le «familial» chez un sujet sans que la famille soit concrètement présente, et donc hors d'un cadre de thérapie familiale au sens strict du terme. Les adolescents qui sont loin de ce travail là peuvent tout de même être entendus sur le versant de leur famille interne et de ce qu'ils en rejouent sur d'autres scènes familiales, ce qui leur permet de reconstruire des représentations imagoïques satisfaisantes.

Le soin ne serait donc pas uniquement un dispositif qui s'articule autour d'une parole curative et d'un travail sur les représentations, mais il s'inscrit parfois bien en amont, dans l'accueil, dans la véritable rencontre, dans le maillage qui s'opère autour de l'adolescent, et dans le travail en réseau institutionnel, ce qui permet de remodeler une enveloppe familiale effractée chez ces sujets en souffrance. Nous pourrions donc parler d'un cadre de soin psychique élargi qui ne se réduit pas au cadre d'entretiens thérapeutiques classiques. 


\section{Cas clinique}

Marie, 16 ans, nous a été adressée il y a deux ans environ par un service de pédopsychiatrie où elle a été hospitalisée pour scarifications. Elle est reçue par une éducatrice spécialisée qui restera sa référente tout au long de ce parcours tumultueux qu'elle aura réalisé dans notre institution de soins aux adolescents. Elle sera prise en charge par le pédopsychiatre du service, à raison d'une séance par quinzaine. Il lui est signifié qu'elle pourra venir à des moments où elle ne va pas bien, et rencontrer l'éducatrice référente si elle est disponible. Au cours de ce parcours elle sera par trois fois réhospitalisée en pédopsychiatrie.

Marie est une grande jeune fille d'origine africaine, arrivée à Lyon lorsqu'elle avait 5 ans et demi, en compagnie de sa mère avec qui elle vit. Ce qui m'interpelle, ce sont ses origines familiales confuses dont elle ne sait pratiquement rien. Ses parents se sont séparés à sa naissance. Elle ne sait pas où est son père, et aux dires de sa mère il serait mort. Elle a des grands parents qui sont restés au pays où elle n'est jamais retournée. Les raisons de son émigration en France restent un mystère, sa mère ne lui en dévoilant rien. Elle a une tante qui habite la région parisienne, une sœur de sa mère.

Mère et fille, très isolées, sont liées par des liens symbiotiques qui les empêchent de vivre l'une sans l'autre, tout en se repoussant violemment parfois, sur le mode de la position narcissique paradoxale (Caillot et Decherf, 1982). Marie a des idées noires avec des bouffées d'angoisse. Elle se scarifie régulièrement, et dit que le sang qui coule la calme. Bien qu'elle ait des amis, elle a du mal à sortir de chez elle, préoccupée par sa mère qui est de santé fragile. Elle est en échec scolaire, ce qui l'a conduite à abandonner le cursus scolaire classique pour s'engager dans une formation professionnelle qui se passe relativement mal, car elle est souvent absente et veut tout arrêter.

Anne, l'éducatrice, reçoit régulièrement Marie qui la sollicite lorsqu'elle va mal. Les rendez-vous avec le médecin sont plus ou moins bien respectés mais ce dernier finit par tolérer ses absences et la laisse investir ce lieu et le travail thérapeutique dont elle bénéficie, à sa façon et à son rythme. Même lorsqu'elle n'a pas rendez-vous, Marie passe des journées entières dans la salle d'attente du service, devant l'écran de l'ordinateur qui est à disposition du public. Sa présence nous interpelle, d'autant plus qu'elle n'a souvent pas de demande précise quand elle vient. Nous nous entendons en équipe sur le fait que sa présence même a valeur de demande implicite, d'autant plus qu'elle ne peut pas investir un lien dans la continuité, se protégeant sans doute d'angoisses d'abandon. Ce qui fait continuité, c'est la manière dont nous l'inves- 
tissons et le sens que nous mettons sur ses présences-absences. Elle justifie ses visites fréquentes par le fait que, n'ayant pas les clefs de chez elle, elle attend que sa mère rentre de son travail.

Marie est régulièrement l'objet de nos préoccupations en réunions d'équipe. Même si cette dernière a pu être divisée sur la réponse à apporter à ses visites sans demande, nous nous entendons cependant sur le fait que l'institution est pour elle un lieu protecteur et qu'elle semble utiliser cette «maison» à la fois dans les représentations familiales qu'elle véhicule et comme lieu de soin. Ainsi elle fait partie intégrante du lieu, sa présence régulière et discrète dans la salle d'attente passe presque inaperçue. Parfois en fin de journée, le personnel lui signifie la fermeture du service et Marie s'en va jusqu'au lendemain. Notre service est devenu pour elle un lieu familier, elle a repéré tout son personnel, et lorsqu'un nouveau arrive, elle interroge Anne pour savoir qui il est. Parfois lorsque nous ne la voyons pas de quelques jours, nous nous inquiétons et en parlons en équipe ou dans des lieux interstitiels. Nous remarquons que Marie occupe nos conversations et focalise sur elle notre souci.

Nous remarquons qu'elle s'infiltre dans les fragilités de l'institution. Son arrivée a coïncidé à un an près avec l'ouverture de ce service. Elle nous pousse à nous interroger sur nos limites et sur les spécificités de chaque professionnel qui y travaille et la complémentarité des uns par rapport aux autres, puisque l'équipe est pluridisciplinaire. Progressivement la malléabilité de l'équipe lui a permis de se reconstruire des représentations plus acceptables et rassurantes à partir de notre propre famille de professionnels.

Nous avons remarqué que les hospitalisations de Marie ont coïncidé en miroir avec celles de sa mère pour dépression. Il est arrivé aussi que la mère aille chercher sa fille et parte avec elle après avoir signé une décharge. Le collage mère-fille rend le soin tel que le souhaiterait l'équipe, difficile pour Marie.

À un moment donné cette dernière, que j'avais eu l'occasion de recevoir ${ }^{1}$ une fois ou deux, alors qu'elle était venue dans notre service lors de bouffées d'angoisses et d'idées suicidaires, a voulu continuer le suivi avec moi et l'arrêter avec le psychiatre. Après en avoir fait part à l'équipe, il a été convenu que je lui signifie qu'elle devait continuer avec lui. A plusieurs reprises cependant, elle a renouvelé auprès de Anna l'éducatrice sa demande. Nous avons fini, en équipe, par accepter qu'elle nous «utilise», à condition de faire du lien entre nous. Ainsi résistons-nous à l'épreuve de la destructivité que Marie agit sur l'équipe autour de la rivalité fraternelle qu'elle activerait entre le psychiatre 
et moi-même (mon collègue psychiatre avançait l'idée qu'elle nous userait les uns après les autres sans rien en symboliser, tandis que j'avais un point de vue contraire). Nous savons à ce moment qu'elle ne se scarifie plus et qu'elle peut venir chez nous demander de l'aide lorsqu'elle va vraiment mal.

J'ai donc signifié à Marie que j'acceptais de la recevoir mais que je continuerais à partager avec l'équipe à propos du souci commun que nous nous faisions pour elle. Marie vint plus ou moins régulièrement à ses rendez-vous, sans forcément toujours prévenir de ses retards ou de ses absences, tandis qu'il lui arrivait aussi de rencontrer de temps en temps son éducatrice référente, véritable figure parentale pour elle. Lorsqu'il arrivait que la mère vienne dans notre service pour apporter les clefs de leur domicile à sa fille, je trouvais toujours le temps de la saluer et d'échanger avec elle quelques mots, sans que je puisse lui faire accepter un entretien mère/fille. La mère refusait de voir qui que ce soit, mais prenait contact avec nous «à la périphérie», avec souvent le prétexte des clefs d'appartement, tout comme sa fille venait assez régulièrement utiliser l'ordinateur sans rien demander d'autre, tout en étant très présente à ce qui se passait autour d'elle.

Durant toute une période, Marie qui était fan d'un groupe pop rock très célèbre auprès des adolescents, me racontait ses voyages au gré des tournées de ce groupe en France et en Allemagne, pays dont les musiciens qui le composent sont originaires, et qui ont la particularité de vivre ensemble et de ne jamais se quitter, d'après elle. À travers ce groupe, Marie interrogeait ainsi le lien familial symbiotique, les relations fraternelles incestuelles, l'ambiguïté des genres, l'homosexualité et les angoisses de séparation. Ce groupe semblait figurer pour Marie une scène familiale dominée par l'indifférenciation, où le regard extérieur des groupies tentait de pénétrer pour savoir ce qu'il se passait vraiment chez eux, telle une scène primitive inquiétante et excitante. J'entendais ainsi comment se déplaçait sur une autre scène une problématique familiale qui pouvait appartenir à Marie (mère et fille collées à la maison) et qu'elle abordait par ces musiciens interposés. En même temps, Marie interrogeait la manière dont on se protège de l'extérieur, et faisait part aussi de manière récurrente de son angoisse que ce groupe se sépare un jour. En tout cas ces déplacements étaient pour Marie l'occasion de partir avec ses copines et de s'éloigner de sa mère.

J'ai également appris qu'elle écrivait des nouvelles, en collaboration avec l'une de ses amies, et qu'elle aimerait faire publier. Ces nouvelles évoquent les aventures d'un personnage imaginaire, mi-homme, mi-femme, sur le modèle de l'un des chanteurs du groupe. Elle confia un jour à l'éducatrice qu'elle uti- 
lisait l'ordinateur du service pour écrire ces histoires imaginaires qu'elle enregistrait ensuite sur une clef USB. Elle utilisait donc l'institution comme un espace transitionnel, pour mettre en imaginaire et en mots, de manière métaphorique, sa propre histoire faite de non-dits et de fantômes. Dans les entretiens, Marie venait me toucher par sa capacité de résilience. Un jour que nous l'avions reçue avec Anne l'éducatrice, elle nous fit part du fait que nous étions un peu pour elle comme des parents.

Elle fit progressivement par la suite le projet de reprendre sa scolarité dans un lycée français en Allemagne, le pays d'origine du groupe pop rock qu'elle utilisait comme modèle et scène à propos des questions sous-jacentes aux impensés de sa propre famille. L'équipe l'a soutenue dans son projet, comportant l'inscription dans un lycée français et la recherche d'une famille d'accueil. Nous l'avons revue juste avant son départ. Elle nous fit part de son anxiété, sa mère lui ayant dit qu'elle serait fière d'elle si elle reprenait une scolarité normale. Nous n'avons plus revu Marie depuis pratiquement un an, mais elle a repris rendez-vous avec moi, pendant les vacances scolaires de février, après avoir vu son éducatrice. Au moment où je faisais part à cette dernière qu'elle n'avait pas honoré son rendez-vous, j'ai appris qu'elle était repartie en Allemagne et qu'elle avait changé de famille d'accueil, reprochant à l'ancienne de trop s'exprimer en français, sa langue «maternelle».

\section{L'écoute groupale}

L'écoute groupale est plus communément utilisée dans le cadre d'une thérapie familiale et de son dispositif spécifique. Il s'agit alors dans le lien transféro-contre-transférentiel de recueillir et de contenir des éléments archaïques qui se manifestent dans l'infra-verbal, à savoir dans ce que la famille donne à voir, à entendre, à ressentir et à sentir. Il s'agit pour les co-thérapeutes de loger en eux une violence provenant de la famille et d'en faire leur intime pour pouvoir la détoxiquer et y mettre du sens. A ce titre, Roman (2001) propose une lecture intéressante du contre-transfert. Le travail ne se fera pas sur les représentations véhiculées par la famille à partir d'un discours secondarisé, mais sur les éprouvés du lien, dans la mesure où les éléments bruts surgissant en séances sous forme de non-dits et d'impensables transgénérationnels, vont se manifester dans un en deçà du discours, en amont de toute forme d'élaboration, et vont faire effraction dans la chaîne associative groupale. Ces éléments bruts sont alors des résurgences de cette "psyché pure » qu'évoquait Ruffiot (1981), en tant que trame psychique inconsciente du groupe familial marquée par le transgénérationnel. 
L'écoute groupale, qui peut aussi se déployer dans le cadre d'une prise en charge individuelle, permet de mettre en évidence la manière dont Marie " parle» de sa famille interne par le fait de diffracter ses groupes internes sur des lieux et des personnages, imagos familiales de substitution, sur lesquels elle dépose des angoisses liées à ses origines confuses qu'elle ne peut symboliser ni verbaliser. Ainsi ces familles imaginaires que représentent entre autres l'institution, le groupe pop-rock, les familles d'accueil en Allemagne (etc.), vont recueillir à la fois ses angoisses autour de l'abandon et de la mort, de l'inceste fraternel, de la confusion des genres, provenant de ses racines identitaires familiales qui ne lui permettent pas, dans un premier temps, de trouver sa place ni une assignation dans le cour de l'adulte.

Par le concept de groupe interne, Kaës $(1993 ; 2005)$ désigne des schèmes de relations d'objet intériorisés qui se réactivent dans le processus groupal, le groupe étant la scène qui permet l'externalisation de formations et de processus psychiques qui appartiennent aux sujets qui le composent. Pour l'auteur, la vie psychique se construit dans les liens et la matrice du groupe primaire (Kaës, 2005). Il situe donc bien le groupe familial comme étant à l'origine de schèmes de liens parfois aliénants, que le sujet réactive par la diffraction sur des groupes externes qu'il est amené à côtoyer. Nous pourrions considérer qu'il s'agirait d'une manière de se libérer de son noyau mélancolique, relatif à des deuils non faits dans l'histoire familiale et qui lui a été transmis à son insu (Durastante, 2009), par le fait de le diffracter sur l'entourage afin de le mettre à l'extérieur sur plusieurs lieux et personnes, pour en éviter tant bien que mal l'impact mortifere et les angoisses afférentes. Les groupes externes pourraient constituer à ce titre des scènes de réactualisations et de figuration pour les irreprésentables traumatiques. Les conduites suicidaires de Marie semblent s'apaiser lorsqu'elle peut rejouer ses angoisses d'abandon sur la scène institutionnelle, ou lorsqu'elle peut déplacer sur le groupe pop rock des problématiques familiales incestueuses relatives à des liens indifférenciés donnant lieu à des places confuses.

L'écoute groupale permet d'entendre comment Marie reconstruit des liens familiaux imaginaires à partir d'autres scènes familiales. Par exemple, sa fréquentation de familles d'accueil en Allemagne met en valeur le fait qu'elle a été capable d'en changer et de choisir la famille qui lui convenait le mieux car elle l'éloignait un peu plus de sa langue «maternelle».

Si Marie n'en était pas à investir un cadre thérapeutique proprement dit autour d'une prise en charge régulière, elle a tout de même bénéficié d'un dispositif soignant dans le travail que nous avons mis en place autour d'un portage «suffisamment bon». 


\section{La véritable rencontre}

Nous allons tenter à présent de comprendre les spécificités de la rencontre thérapeutique avec Marie. Il s'est agi de l'accueillir là où elle en était, à son rythme propre, et de se laisser toucher et transformer par cette rencontre qui est avant tout une rencontre humaine, de la même manière que Marie s'est laissé toucher et transformer par l'authenticité de la rencontre thérapeutique.

Duteille (2002) fait part du caractère inaugural de toute rencontre authentique, susceptible de transformer profondément le sujet, et de manière radicale, selon un point de vue socio-anthropologique. L'auteur souligne que ce type de rencontre n'est pas fortuit, mais inconsciemment attendue par le sujet qui est «rencontré par ce qu'il poursuit» (ibidem, p. 83). Ce qui distingue la rencontre d'une interaction ordinaire c'est le fait qu'elle permet au sujet de se reconstruire, et de rompre la continuité du vécu quotidien pour permettre d'accéder à d'autres aspects de soi par la médiation de l'autre. L'auteur avance que ces rencontres exceptionnelles donnent lieu à des ruptures temporelles non pathologiques qui s'insinuent dans un temps quotidien linéaire, constitué par plusieurs mondes (donnant lieu à des modes de vécu différents) qui s'interpénètrent (réalité concrètement perçue, rêve, imaginaire, etc.). Ce point de vue phénoménologique contient l'idée que ce type de rencontre, en même temps qu'elle donne naissance à des liens nouveaux, fait nécessairement rupture, dans la mesure où elle déclenche un désordre qui annonce un nouvel ordre. La rencontre ouvre le sujet à la créativité, dans la mesure où elle incarne le lieu du possible.

Gutton (2008) insiste sur le fait que l'adolescent invente ce qu'il est et qui il est. Il m'a semblé que cette créativité ressort dans la manière dont l'adolescent se confronte à des expériences, parfois extrêmes afin de se construire. Au cours de celles-ci, où il interpelle fortement l'adulte, il peut, tout comme Marie, faire des rencontres initiatiques d'adultes qui portent sur elle un regard valorisant lui permettant de réaménager sa groupalité interne. Pour B. Cyrulnik (2003), le tuteur de résilience est cette rencontre qui permet au sujet de sublimer son mal-être et d'en faire un processus créatif. Nous dirions pour notre part que la rencontre de Marie avec notre institution, notre capacité de contenance et le regard valorisant que nous lui portions mettant en valeur notre croyance en ses capacités à changer et à transformer sa vie, lui ont permis de mettre du sens sur les liens nouveaux qu'elle établissait et qui nous amenaient à modifier ceux qui avaient cours dans notre institution. La véritable rencontre est faite de cette capacité de part et d'autre à se laisser trans- 
former par l'autre. Dans le contre-transfert, Marie me touchait par sa capacité à se reconstruire, par sa mobilité psychique qui l'amenait à cet acte de création qu'est l'écriture, par la «débrouillardise» dont elle faisait preuve lors de ses voyages et aussi pour construire son projet de re-scolarisation à l'étranger. Cette rencontre m'a permis d'affiner mes constructions théorico-cliniques à propos du lien thérapeutique avec l'adolescent. Je me suis donc moi aussi laissé toucher et transformer par cette rencontre.

\section{Le dispositif en tuilage}

Lors de mes travaux de recherche et dans ma pratique clinique, j'ai fait de cette modalité du cadre sur mesure, que j'ai nommé «dispositif en tuilage », un outil de travail dans les prises en charge d'adolescents et de leur famille (Durastante, 2009, 2011; Joubert et Durastante, 2008).

\section{Disposition interne et dispositif en tuilage}

La disposition interne contient l'idée d'être disponible pour quelqu'un, à son service, c'est à dire à son rythme, d'être intuitivement en contact avec lui et donc de pouvoir s'engager dans une relation suffisamment familière pour s'approcher ou pour se laisser approcher assez près, afin de permettre à l'adolescent de jouer avec des limites malléables, dans le lien thérapeutique.

Notre pratique au quotidien avec les adolescents me donne à penser que ce qui est soignant c'est aussi le lien, un lien suffisamment sécure qui peut être tissé autour d'eux. C'est dans cet objectif que j'ai nommé « dispositif en tuilage» un cadre sur mesure tenant compte du rythme du patient. Le tuilage évoque le bâtir d'une toiture en tant qu'élément essentiel d'une maison, mon approche étant avant tout groupale-familiale. Mais le tuilage évoque aussi une technique de chant polyphonique où chaque chanteur reprend les dernières paroles du chanteur qui le précède. Cela évoque deux idées maîtresses de ce dispositif: la contenance et le tissage de liens intersubjectifs avec l'idée de jouer à plusieurs sur la même partition. Le dispositif en tuilage a été pensé pour aider l'adolescent à se resituer à une place spécifique dans son histoire générationnelle à partir du moment où le maillage de liens inter-partenariaux qui s'opère sur la cité, à partir du souci commun que nous nous faisons pour tel ou tel jeune et sa famille, l'inscrit dans une enveloppe familiale imaginaire à l'endroit où la sienne propre a été effractée. Les éléments essentiels du dispositif en tuilage sont la suffisante familiarité, la malléabilité et le travail partenarial en réseau. 


\section{Suffisante familiarité et malléabilité}

Ces valeurs comportent le fait d'être proche d'un patient sans pour cela transgresser la règle d'abstinence. Une familiarité bien tempérée permet d'activer des processus d'identification à partir du moment où le patient retrouve chez le thérapeute un peu de «même», par le fait d'être accueilli avec humanité et chaleur, ce qui renvoie ce patient à une position de sujet.

La malléabilité favorise la prise en compte de la relation transféro-contretransférentielle qui permet au soignant de se sentir suffisamment contenant pour supporter d'être envahi d'affects pénibles autour de l'absence, de l'abandon et du manque provoqué par le comportement en rupture du jeune au fil du suivi thérapeutique. Cette tolérance à l'absence et cette certitude que malgré tout le clinicien reste à sa disposition, ouvre chez l'adolescent un espace de liberté et de créativité, dans un dispositif dont il n'est pas prisonnier et qui le respecte dans son rythme propre, à partir de l'assurance qui lui est faite que ce professionnel n'a pas été détruit par la recherche de limites et de contenance que l'adolescent lui oppose souvent (la tolérance de l'équipe à la manière dont Marie a investi l'institution a permis à cette dernière d'exprimer sa créativité). Tout au long du suivi de Marie, nous avons pu penser en équipe ses absences comme autant de demandes sous-jacentes d'être rejointe dans ce qu'elle mettait en scène de l'abandon.

\section{Le travail partenarial en réseau}

Cette «famille» imaginaire, que constitue notre institution a sans doute constitué pour Marie l'exemple même d'adultes capables de s'entendre à propos d'elle et de la faire exister en tant que sujet dans une dynamique de réseau. L'expérience de ce maillage est susceptible à la longue d'être introjectée par la patiente, lui proposant par là même une place dans une famille imaginaire, ce qui a pour effet de l'aider à tisser des liens externes et internes avec la sienne propre. Ainsi l'introjection progressive des liens qui se tissent avec les adultes qu'elle rencontre lui permet-elle de modifier ses représentations du lien familial, afin de pouvoir se reconnaître en tant que membre d'une groupalité familiale imaginaire et de se réconcilier par là même avec sa propre famille. Le travail partenarial permet d'amener une réflexion sur les spécificités de chacun, et de resituer chacun à une place complémentaire de celle des autres. Marie nous fait part à un moment donné que nous représenterions pour elle, l'éducatrice et moi-même, des parents imaginaires qui l'investissent et pour qui elle compte. Cet imaginaire partagé permet un remodelage des groupes internes, dans l'espace intrapsychique de l'adolescente, par l'introjection 
d'imagos familiales satisfaisantes, à partir du moment où elle-même se sent digne d'être investie.

\section{Reconstruction imagoïque et objet transgénérationnel}

Ce concept d'imago pourrait faire référence à ce qu'écrivait A. Ruffiot (1981) sur le fait que les imagos inconscientes constituent un organisateur du groupe familial. Elles ont une fonction régularisatrice tant sur le plan intrapsychique que dans les interactions familiales. Il faut aussi évoquer le concept d'objet transgénérationnel développé par A. Eiguer (1997). L'idée centrale que nous proposons, est que l'objet transgénérationnel, véritable entité fantasmatique à propos d'un ancêtre ayant marqué les générations antérieures, et qui se déploie ou pas dans le discours des parents, permet à l'enfant et à l'adolescent de se décentrer et d'avoir accès dans le meilleur des cas aux imagos qui lui permettent de reconstituer d'autres possibles, sur le plan fantasmatique, et de se relier ainsi progressivement à l'ordre des générations.

L'on peut penser que toute rencontre que va faire l'enfant et l'adolescent, va lui permettre, à travers le lien qu'il établit avec d'autres sujets extérieurs à la famille, de se construire des imagos parentales et/ou grand-parentales acceptables, et de redonner forme à un objet transgénérationnel de substitution, lorsque ce dernier a été défaillant dans le discours des parents, ce qui ne lui a pas permis de se relier fantasmatiquement à l'ordre des générations. Ainsi le soignant, via la dynamique transféro-contre-transférentielle, peut figurer pour un adolescent une imago parentale et/ou grand-parentale acceptable, et les liens qui s'établissent entre soignants, comme autant d'imagos familiales reliées entre elles et qui le «maillent » ainsi dans un dispositif contenant.

\section{Conclusion}

Le sujet singulier, toujours sujet du groupe, est déterminé par ses groupes internes qui relatent la manière dont il porte en lui son groupe primaire ainsi que les modalités de liens qui se sont mises en place et qui lui ont été transmises à son insu. Si l'on fait l'hypothèse que ces dernières sont étroitement liées au transgénérationnel, l'on peut admettre que l'approche soignante doit prendre en compte cette dimension groupale du sujet, qui va renseigner le soignant sur ce que ce dernier dépose de ses souffrances sur d'autres scènes groupales qu'il rencontre dans sa vie. Il s'agit d'une autre manière d'écouter cette famille qui peuple la psyché du sujet et qui en constitue son identité. Le maillage de liens interinstitutionnels qui peut se mettre en place autour de lui 
peut être considéré comme une étape du soin, dans la mesure où l'institution constitue alors une enveloppe familiale contenante, susceptible de l'aider à réaménager ses imagos familiales et à pouvoir se séparer progressivement des siens pour construire sa vie avec une représentation de lui-même enrichie.

\author{
Richard Durastante, \\ 39 Quai Gailleton, 69002 Lyon \\ rdura@wanadoo.fr \\ Christiane Joubert, \\ 3 rue Coysevox Lyon 69001 \\ christianejoubert@netcourrier.com
}

\title{
Note
}

1. L'utilisation du «je» renvoie au premier auteur, et permet d'éviter la confusion entre ce qui est de l'implication d'un soignant et celle de l'équipe.

\section{Références}

CAILLOT, J. P., DECHERF, G., 1982, Thérapie familiale et paradoxalité, Clancier-Guénaud, Paris.

CYRULNIK, B., 2003, Comment un professionnel peut-il devenir un tuteur de résilience?, in Cyrulnik, B., Seron, C., éds., La résilience ou comment renaître de sa souffrance, Fabert, Paris, 23-43.

DURASTANTE, R., 2009, Du transgénérationnel à la mort désavouée : les jeux d'asphyxie, in Morhain, Y., Roussillon, R., éds., Actualités psychopathologiques de l'adolescence, De Boeck, Bruxelles, 143-170.

DURASTANTE, R., 2011, Adolescence et addictions. De la crypte familiale au dispositif en tuilage, De Boeck, Bruxelles.

DUTEILLE, C., 2002, L'événement de la rencontre comme expérience de rupture temporelle, Arobase, 6, 1-2, 81-88.

EIGUER, A., 1997, La part maudite en héritage, in Eiguer, A., et al., éds., Le générationnel. Approche en thérapie familiale psychanalytique, Dunod, Paris, 2002, 13-68.

GUTTON, P., 2008, Le génie adolescent, Odile Jacob, Paris.

JOUBERT, C., DURASTANTE, R., 2008, Le cadre en tuilage : place et reconnaissance de l'adolescent en famille, Le Divan Familial, 21, 67-80.

KAËS, R., 1993, Le groupe et le sujet du groupe, Dunod, Paris.

KAËS, R., 2005, Groupes internes et groupalité psychique : genèse et enjeu d'un concept, Revue de psychothérapie psychanalytique de groupe, 2, 45, 9-30.

ROMAN, P., 2001, La violence dans le contre-transfert, Souffles, 162, 18-25.

RUFFIOT, A., 1981, La thérapie familiale psychanalytique, Dunod, Paris. 